Author affiliations appear at the end of this article.

Published online ahead of print at www.jco.org on September 14, 2015.

Support information appears at the end of this article.

Presented in part at the 56th Annual Meeting of the American Society of Hematology, San Diego, CA, December $6-9,2014$

The views expressed in this article do not reflect the official policy or position of the National Institutes of Health, the Department of the Navy, the Department of Defense, the Health Resources and Services Administration, or any other agency of the US Government.

Authors' disclosures of potential conflicts of interest are found in the article online at www.jco.org. Author contributions are found at the end of this article.

Corresponding author: Anita D'Souza, MD, Division of Hematology Oncology, Department of Medicine, Medical College of Wisconsin 9200 W Wisconsin Ave, Ste C5500, Milwaukee, WI 53226; e-mail: andsouza@mcw.edu.

(C) 2015 by American Society of Clinical Oncology

0732-183X/15/3332w-3741w/\$20.00 DOI: 10.1200/JCO.2015.62.4015

\title{
Improved Outcomes After Autologous Hematopoietic Cell Transplantation for Light Chain Amyloidosis: A Center for International Blood and Marrow Transplant Research Study
}

Anita D’Souza, Angela Dispenzieri, Baldeep Wirk, Mei-Jie Zhang, Jiaxing Huang, Morie A. Gertz, Robert A. Kyle, Shaji Kumar, Raymond L. Comenzo, Robert Peter Gale, Hillard M. Lazarus, Bipin N. Savani, Robert F. Cornell, Brendan M. Weiss, Dan T. Vogl, César O. Freytes, Emma C. Scott, Heather J. Landau, Jan S. Moreb, Luciano J. Costa, Muthalagu Ramanathan, Natalie S. Callander, Rammurti T. Kamble, Richard F. Olsson, Siddhartha Ganguly, Taiga Nishihori, Tamila L. Kindwall-Keller, William A. Wood, Tomer M. Mark, and Parameswaran Hari

See accompanying editorial on page 3689

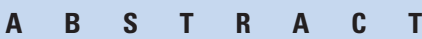

\section{Purpose}

Autologous hematopoietic cell transplantation, or autotransplantation, is effective in light-chain amyloidosis $(A L)$, but it is associated with a high risk of early mortality $(E M)$. In a multicenter randomized comparison against oral chemotherapy, autotransplantation was associated with $24 \%$ EM. We analyzed trends in outcomes after autologous hematopoietic cell transplantation for AL in North America.

\section{Patients and Methods}

Between 1995 and 2012, 1,536 patients with AL who underwent autotransplantation at 134 centers were identified in the Center for International Blood and Marrow Transplant Research database. EM and overall survival (OS) were analyzed in three time cohorts: 1995 to 2000 ( $\mathrm{n}=$ 140), 2001 to 2006 ( $n=596$ ), and 2007 to 2012 ( $n=800)$. Hematologic and renal responses and factors associated with EM, relapse and/or progression, progression-free survival and OS were analyzed in more recent subgroups from 2001 to $2006(n=197)$ and from 2007 to 2012 ( $n=157$ ).

\section{Results}

Mortality at 30 and 100 days progressively declined over successive time periods from $11 \%$ and $20 \%$, respectively, in 1995 to 2000 to $5 \%$ and $11 \%$, respectively, in 2001 to 2006, and to $3 \%$ and 5\%, respectively, in 2007 to 2012 . Correspondingly, 5-year OS improved from 55\% in 1995 to 2000 to $61 \%$ in 2001 to 2006 and to $77 \%$ in 2007 to 2012 . Hematologic response to transplantation improved in the latest cohort. Renal response rate was 32\%. Centers performing more than four AL transplantations per year had superior survival outcomes. In the multivariable analysis, cardiac AL was associated with high EM and inferior progression-free survival and OS. Autotransplantation in 2007 to 2012 and use of higher dosages of melphalan were associated with a lowered relapse risk. A Karnofsky score less than 80 and creatinine levels $2 \mathrm{mg} / \mathrm{m}^{2}$ or greater were associated with worsened OS.

\section{Conclusion}

Post-transplantation survival in $\mathrm{AL}$ has improved, with a dramatic reduction in early posttransplantation mortality and excellent 5-year survival. The risk-benefit ratio for autotransplantation has changed, and randomized comparison with nontransplantation approaches is again warranted.

J Clin Oncol 33:3741-3749. (C) 2015 by American Society of Clinical Oncology

\section{INTRODUCTION}

Light-chain amyloidosis (AL) is a multisystemic disease caused by a malignant plasma cell clone with resultant insoluble fibrillar deposition. ${ }^{1}$ Amyloid fibrils derived from misfolded immunoglobulin light chains cause direct organ toxicity leading to organ failure and death. Current AL therapies focus on eliminating the source of amyloid, that is, the plasma cell clone, by using chemotherapy. However, che- motherapy has minimal effect on preformed organ amyloid; consequently, organ improvements lag behind hematologic responses. ${ }^{2}$ After diagnosis, patients, particularly those with advanced cardiac AL, are at increased risk for early death from progressive heart failure and sudden death despite the use of effective chemotherapy. ${ }^{3}$

The role of autologous hematopoietic cell transplantation, or autotransplantation, in $\mathrm{AL}$ is controversial. Single-center studies revealed good 
outcomes in terms of hematologic responses and organ improvement. ${ }^{4-6}$ However, the prospective randomized clinical trial reported in 2007 in which autotransplantation was compared with oral melphalan and dexamethasone showed inferior survival with autotransplantation. ${ }^{7}$ Inferior outcomes were related to high early mortality (EM) of $24 \%$ in the transplantation arm and were partly attributed to the inclusion of patients with severe cardiac amyloidosis $^{7}$ and possibly related to low-volume amyloid transplantation centers. ${ }^{8}$ Since that study, ${ }^{7}$ use of cardiac biomarkersserum troponin $\mathrm{T}$ and $\mathrm{N}$-terminal of brain natriuretic peptide - has helped improve detection and risk stratification in patients with cardiac AL. 9 These refined selection criteria for autotransplantation, along with improvements in supportive care of patients in the peritransplantation period, have translated into a decrease in the post-transplantation EM to less than 5\% at large amyloid centers in the United States. ${ }^{10,11}$ We hypothesized that outcomes of autotransplantation among patients with AL have improved over time. In addition, we wanted to test the utility of autotransplantation in $\mathrm{AL}$ in practice and to assess if center $\mathrm{AL}$ transplantation experience affects outcomes. We undertook a retrospective study using the Center for International Blood and Marrow Transplant Research (CIBMTR) database to address these issues.

\section{PATIENTS AND METHODS}

\section{Data Source}

The CIBMTR is a prospectively maintained registry that collects transplantation data from over 320 centers worldwide. Data are collected at two levels: registration and research. The registration data include disease type, age, sex, date of diagnosis, graft type, conditioning regimen, post-transplantation disease progression, survival, and cause of death, and it includes all transplantations reported to the CIBMTR. More-detailed clinical data are collected from a subgroup of registered patients selected for research data by using a weighted randomization scheme. Both the registration data and the research data are collected pretransplantation, at 100 days and 6 months post-transplantation, and annually thereafter until death or last follow-up.

\section{Patients}

Included in this study were patients registered with the CIBMTR in North America between 1995 and 2012 for an autotransplantation for AL within 24 months of diagnosis. Data from these patients were analyzed in three arbitrarily grouped cohorts based on the year of transplantation: 1995 to 2000, 2001 to 2006, and 2007 to 2012. Registration data set was used to assess survival outcomes. Robust research data were available for a subgroup of 354 patients who underwent transplantation between 2001 and 2012. This subgroup was analyzed for hematologic and organ responses and in multivariable analyses. Key characteristics and survival were compared between the registration set and this subset to confirm that the subgroup was a random representative of the data set (Appendix Table A1, online only, and Appendix Table A2, online only). Data accuracy was established by means of physician review (by A. D'Souza and P.H.) of discrepant data when present, and centers were contacted if needed for additional review.

\section{Definitions of Early Mortality, Response, and Center Effect}

EM was defined as mortality from any cause after transplantation within the defined time windows of 30 and 100 days. This differed from transplantation-related mortality (TRM), which excluded patients who died as a result of progressive AL or relapse. This avoided attribution bias wherein mortality from organ amyloid in the post-transplantation period could have been labeled as being caused by disease and, thus, causing an underestimation of TRM. Use of EM ensured that the risk of post-transplantation death was not underreported and that mortality in the post-transplantation period was accurately described irrespective of attribution to disease versus treatmentrelated causes.

The uniform system proposed at the 10th International Symposium on Amyloidosis was used to define hematologic and renal response and progressions. ${ }^{12}$ In 2012, new criteria were proposed to define hematologic response and progression on the basis of free light-chain (FLC) analysis. ${ }^{3}$ Because FLC and cardiac biomarkers were collected only after 2008, we were unable to apply the 2012 hematologic or cardiac response criteria to our data set retrospectively. Among organ responses, only renal response is reported in this analysis.

The association between amyloid transplantation volume and survival was analyzed as center experience. ${ }^{8}$ Owing to a wide variability in the numbers of transplantations performed by centers over the 18 -year period of this study, center effect was calculated by using the mean number of amyloid transplantations performed per year over the 4 years ${ }^{13}$ between 2009 and 2012. Center experience in transplantation for $\mathrm{AL}$ was considered a dichotomous variable. After we preformed maximum likelihood testing using the various cut points of two, three, four, five, and 10 amyloid transplantations per year, we established that a minimum of four AL transplantations per year was an informative divider for this analysis.

\section{Statistical Analysis}

Patient-, disease- and treatment-related factors were compared by using the $[\mathrm{CHI}]^{2}$ test for categorical variables and the Kruskall-Wallis test for continuous variables. Outcomes analyzed included EM, relapse and/or progression, progression-free survival (PFS), and overall survival (OS). Estimates of outcomes were reported as probabilities with $95 \%$ CIs. The probability of OS was calculated with the Kaplan-Meier estimator, with the variance estimated by using the Greenwood formula. Survival curves were compared with the log-rank test. Multivariable analysis was performed by using the research data subset $(\mathrm{n}=354)$ with a Cox proportional hazards model in which the transplantation period cohort of 2001 to 2006 versus 2007 to 2012 was the main effect. Other factors included age at transplantation, race, pretransplantation Karnofsky performance score (KPS), hematopoietic cell transplantationcomorbidity index (HCT-CI), serum creatinine level, serum albumin level, bone marrow plasma cell burden, amyloid organ pattern, number of involved organs, pretransplantation chemotherapy, time from diagnosis to transplantation, melphalan dose in $\mathrm{mg} / \mathrm{m}^{2}$ and center effect. The assumption of proportional hazards was tested for each variable, and factors violating the proportionality assumption were adjusted by stratification. Potential interactions between the main effect and all other significant risk factors were tested. All $P$ values were two-sided, and $P<0.05$ was considered to indicate a significant difference.

\section{RESULTS}

\section{Patient-, Disease-, and Transplantation- Related Variables}

Table 1 displays available characteristics for the entire group from 1995 to $2012(\mathrm{n}=1,536)$, and Table 2 shows data for the subset with detailed level data from 2001 to $2012(\mathrm{n}=354)$. The median age at transplantation was 56 years, with evidence of increasing age at transplantation with successive time cohorts (Table 1). Most patients underwent transplantation within 6 months of diagnosis. The underlying plasma cell clone was $\lambda$ in $72 \%$ of cases (Table 2). The M-spike was nonquantifiable in $42 \%$ of patients; when quantified, the median size was $0.6 \mathrm{~g} / \mathrm{dL}$. The distribution of cardiac and renal involvement was similar between the groups. Few patients had noncardiac, nonrenal organ involvement in this 


\begin{tabular}{|c|c|c|c|c|}
\hline \multirow[b]{3}{*}{ Characteristic } & \multicolumn{4}{|c|}{ No. (\%) } \\
\hline & \multicolumn{3}{|c|}{ Year of Transplantation } & \multirow[b]{2}{*}{ Overall $(\mathrm{N}=1,536)$} \\
\hline & $1995-2000(n=140)$ & $2001-2006(n=596)$ & $2007-2012(n=800)$ & \\
\hline No. of centers & 50 & 93 & 104 & 134 \\
\hline \multicolumn{5}{|l|}{ Age at transplantation, years } \\
\hline Median age (range) & $54(31-71)$ & $57(23-78)$ & $59(26-77)$ & $56(23-78)$ \\
\hline $18-39$ & $12(9)$ & $21(4)$ & $23(3)$ & $272(18)$ \\
\hline $40-49$ & $30(21)$ & $123(21)$ & $119(15)$ & 604 (39) \\
\hline $50-59$ & $65(46)$ & $244(41)$ & 295 (37) & $362(24)$ \\
\hline $60-65$ & $30(21)$ & $118(20)$ & $214(27)$ & $242(16)$ \\
\hline$\geq 66$ & $3(2)$ & 90 (15) & 149 (19) & $56(4)$ \\
\hline Male sex & $85(61)$ & $355(60)$ & $483(60)$ & $923(60)$ \\
\hline Karnofsky score $<80$ & $21(15)$ & $82(14)$ & $110(14)$ & $213(14)$ \\
\hline \multicolumn{5}{|l|}{ Time from diagnosis to transplant, months } \\
\hline$<6$ & $80(57)$ & $399(67)$ & $483(60)$ & $962(63)$ \\
\hline $6-12$ & $41(29)$ & $145(24)$ & $214(27)$ & $400(26)$ \\
\hline $12-24$ & $19(14)$ & $52(9)$ & $103(13)$ & $174(11)$ \\
\hline Median follow-up of survivors from diagnosis (range), months & $146(7-209)$ & $97(5-152)$ & $33(6-94)$ & $56(5-209)$ \\
\hline Median follow-up of survivors from transplantation (range), months & $142(4-194)$ & $93(3-145)$ & $25(3-76)$ & $49(3-194)$ \\
\hline
\end{tabular}

cohort, and renal involvement without cardiac involvement was the most frequently observed pattern. We found no difference in the 2001-to-2006 and 2007-to-2012 groups in terms of the number of organs involved, and four or more organ were involved in 12\% and $11 \%$ patients, respectively. Most patients had no treatment before transplantation, but more patients received treatment in 2007 to 2012 than in 2001 to 2006 (33\% v 13\%, $P<.001)$. Melphalan dosage was reduced most frequently in 2007 to 2012, with $64 \%$ of patients receiving less than $180 \mathrm{mg} / \mathrm{m}^{2}$ of melphalan conditioning, and $34 \%$ received less than $140 \mathrm{mg} / \mathrm{m}^{2}$.

\section{EM and Causes of Death in 1,536 Patients}

Figure 1A shows EM for the three groups. Mortality at 30 and 100 days declined from $11 \%$ (95\% CI, 7\% to $17 \%$ ) and $20 \%(95 \%$ CI, $14 \%$ to $27 \%$ ), respectively, for the 1995 -to-2000 group to $5 \%$ (95\% CI, $4 \%$ to $7 \%)$ and $11 \%(95 \% \mathrm{CI}, 8 \%$ to $13 \%)$ for the 2001-to-2006 group to $3 \%$ (95\% CI, $2 \%$ to $4 \%$ ) and 5\% (95\% CI, $4 \%$ to $7 \%)$ for the 2007 -to-2012 group $(P<.001)$. No difference was seen in EM based on the time from diagnosis to transplantation. Rates were, at less than 6 months, $12 \%$ (95\% CI, $8 \%$ to $17 \%$ ); at 6 to 12 months, 5 (95\% CI, $1 \%$ to $11 \%$ ); and 12 to 24 months, $8 \%$ ( $95 \%$ CI, $2 \%$ to $18 \%$ ). Causes of death reported within the first 100 days were attributed to amyloid and organ failure in most patients $(83 \%)$, followed by infection ( $8 \%)$, nonengraftment $(3 \%)$, and unknown causes $(6 \%)$.

\section{OS in 1,536 Patients}

Median follow-up was 56 months (5 to 209 months) from the time of diagnosis. One-, 3- and 5-year OS in successive cohorts improved from $75 \%$ ( $95 \% \mathrm{CI}, 65 \%$ to $82 \%$ ), $64 \%$ (95\% CI, $56 \%$ to $72 \%$ ), and $55 \%$ (95\% CI, $46 \%$ to $63 \%$ ), respectively, for 1995 to 2000 to $85 \%$ (95\% CI, $81 \%$ to $87 \%$ ), $72 \%$ (95\% CI, $68 \%$ to $75 \%$ ), and $61 \%(95 \%$ CI, $57 \%$ to $65 \%$ ), respectively, for 2001 to 2006 and to $90 \%$ (95\% CI,
$88 \%$ to $92 \%$ ), $83 \%$ ( $95 \%$ CI, $80 \%$ to $86 \%$ ), and $77 \%$ (95\% CI, 72 to $82 \%)$, respectively, for 2007 to $2012(P<.001$; Fig 1B).

We observed no difference in OS based on the time from diagnosis to transplantation. Rates were, at less than 6 months, 73\% (95\% CI, $67 \%$ to $79 \%$ ); at 6 to 12 months, $81 \%$ (95\% CI, $71 \%$ to $89 \%)$; and at 12 to 24 months, $81 \%(95 \% \mathrm{CI}, 61 \%$ to $92 \% ; P=0.22)$.

For patients with cardiac amyloidosis, 3-year OS improved from $62 \%$ (95\% CI, $48 \%$ to $75 \%$ ) in 2001 to 2006 to $67 \%$ (95\% CI, $52 \%$ to $80 \%)$ in 2007 to $2012(P=.59$; Fig 2A). For those with renal and without cardiac amyloidosis, 3-year OS improved from 78\% (95\% CI, $68 \%$ to $86 \%$ ) in 2001 to 2006 to $89 \%$ (95\% CI, $82 \%$ to $95 \%$ ) in 2007 to $2012(P=.03$; Fig $2 \mathrm{~B})$.

\section{Response Rates in the Subset of 354 Patients}

A best hematologic response to transplantation was available in $89 \%$ and $97 \%$ of patients in the 2001-to-2006 and 2007-to-2012 groups, respectively. A renal response at any point after autotransplantation was available in $70 \%$ and $78 \%$ of patients in 2001 to 2006 and 2007 to 2012, respectively. Table 3 shows a breakdown of hematologic and renal responses that were observed in this cohort. The hematologic response rate was higher in the 2007-to-2012 cohort than in the 2001-to-2006 cohort.

\section{Center Effects in the Subset of 354 Patients}

EM was worse among 81 centers that performed fewer than four AL transplantations per year, that is, low volume, than in others. Their mortality rate of $5 \%(95 \% \mathrm{CI}, 3 \%$ to $7 \%)$ at 30 days and $7 \%(95 \% \mathrm{CI}$, $5 \%$ to $10 \%)$ at 100 days compared with $1 \%(95 \% \mathrm{CI}, 0.4 \%$ to $3 \%)$ at 30 days and $3 \%(95 \%$ CI $2 \%$ to $6 \%$ ) at 100 days for centers that performed four or more AL transplantations a year, or high volume (n $=11 ; P=.01 ;$ Fig $2 \mathrm{C}$ ). No statistically significant difference was found for age, KPS, HCT-CI, cardiac amyloidosis, number of organs 
Table 2. Baseline Characteristics of Patients From 2001-2012 Subgroup

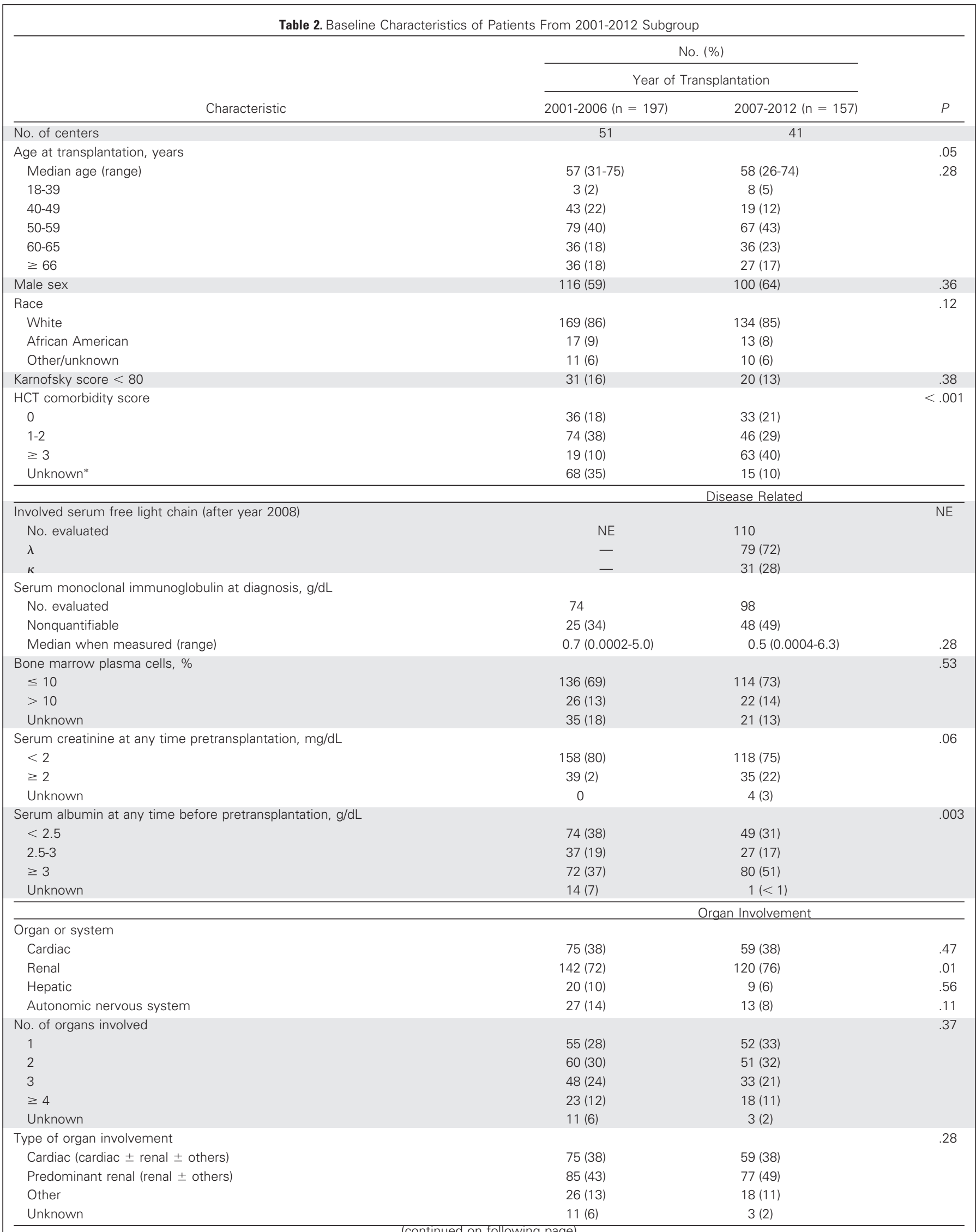




\begin{tabular}{|c|c|c|c|}
\hline \multirow[b]{3}{*}{ Characteristic } & \multicolumn{2}{|c|}{ No. $(\%)$} & \multirow[b]{3}{*}{$P$} \\
\hline & \multicolumn{2}{|c|}{ Year of Transplantation } & \\
\hline & $2001-2006(n=197)$ & $2007-2012(n=157)$ & \\
\hline & \multicolumn{3}{|c|}{ Transplantation Related } \\
\hline CD34 cells infused, $\times 10^{6} / \mathrm{kg}$ & 160 & 113 & .01 \\
\hline Median (range) & $4.8(1.0-16.8)$ & $4.0(1.2-17.3)$ & \\
\hline Melphalan dose, $\mathrm{mg} / \mathrm{m}^{2}$ & & & .01 \\
\hline Median (range) & 175 (86-241) & 143 (86-219) & .15 \\
\hline$<140$ & $65(33)$ & $69(44)$ & \\
\hline $140-180$ & $31(16)$ & $31(20)$ & \\
\hline$\geq 180$ & $86(44)$ & $54(34)$ & \\
\hline Missing/outliers & $15(8)$ & $3(2)$ & \\
\hline Pretransplantation chemotherapy & & & $<.001$ \\
\hline Untreated & $167(85)$ & $105(67)$ & \\
\hline Melphalan based & $13(7)$ & $15(10)$ & \\
\hline IMID based & $12(6)$ & $22(14)$ & \\
\hline Bortezomib based & 0 & $15(10)$ & \\
\hline Missing & $5(3)$ & 0 & \\
\hline Time from diagnosis to transplantation, months & & & .92 \\
\hline$<6$ & $131(66)$ & $103(66)$ & \\
\hline $6-12$ & $46(23)$ & $36(23)$ & \\
\hline $12-24$ & $20(10)$ & $18(11)$ & \\
\hline Median follow-up of survivors from diagnosis (range), months & $89(5-51)$ & $56(12-82)$ & \\
\hline Median follow-up of survivors from transplant (range), months & $77(3-145)$ & $49(7-75)$ & \\
\hline
\end{tabular}

involved with amyloidosis, melphalan conditioning dosage, or pretransplantation chemotherapy between the high-volume and lowvolume centers (Appendix Table A3, online only). Both high- and low-volume centers showed an improvement in reported EM in the three time cohorts $(P<.01$; Fig 2D).

\section{Multivariable Analysis in the Subset of 354 Patients}

Table 4 shows results of the multivariable analysis. Adjusted analysis showed no difference between the 2001-to-2006 cohort and the 2007-to-2012 cohort for EM, PFS, or OS. The analysis for relapse and/or progression was nonproportional, and the optimal cut point
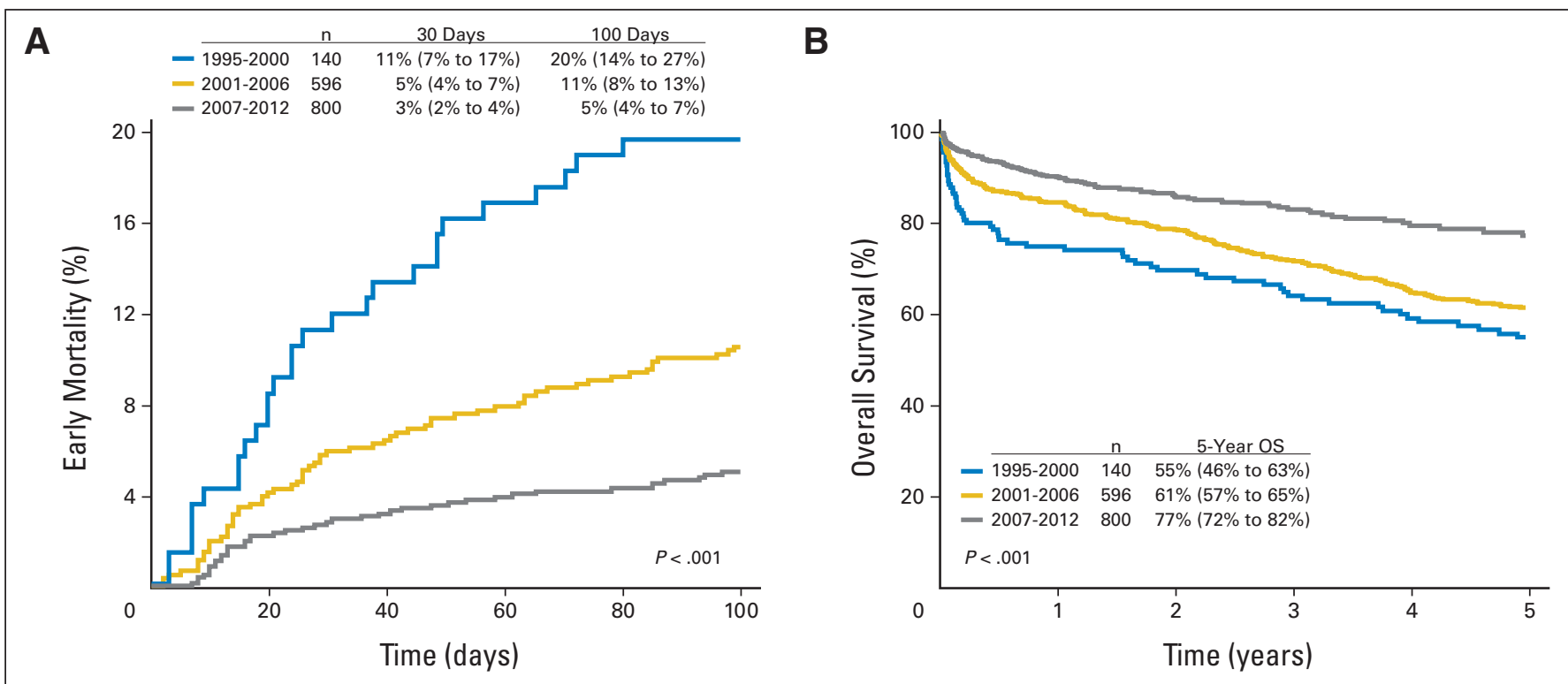

Fig 1. Probabilities of (A) early mortality and (B) overall survival (OS) in light-chain amyloidosis after transplantation for all three time cohorts. 


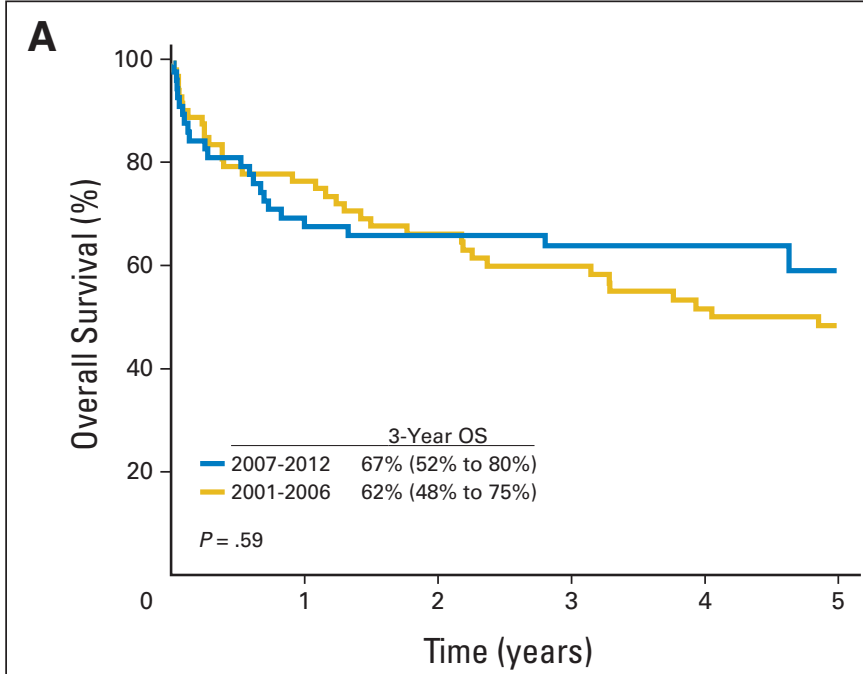

B

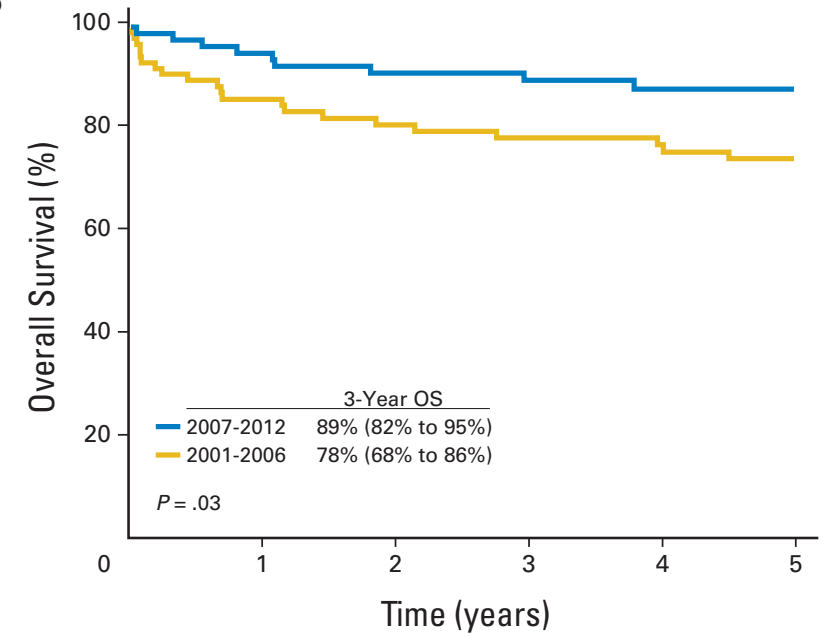

C

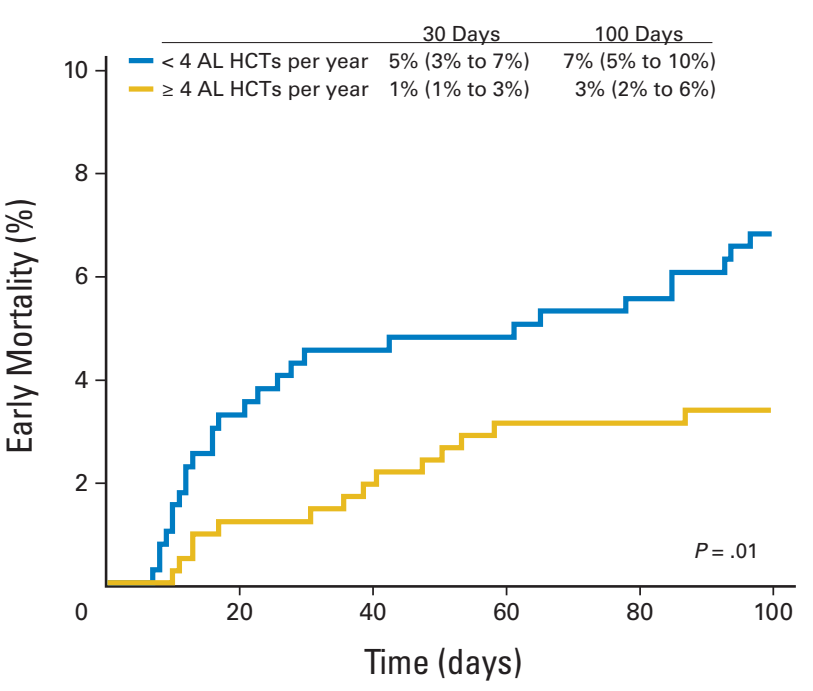

D

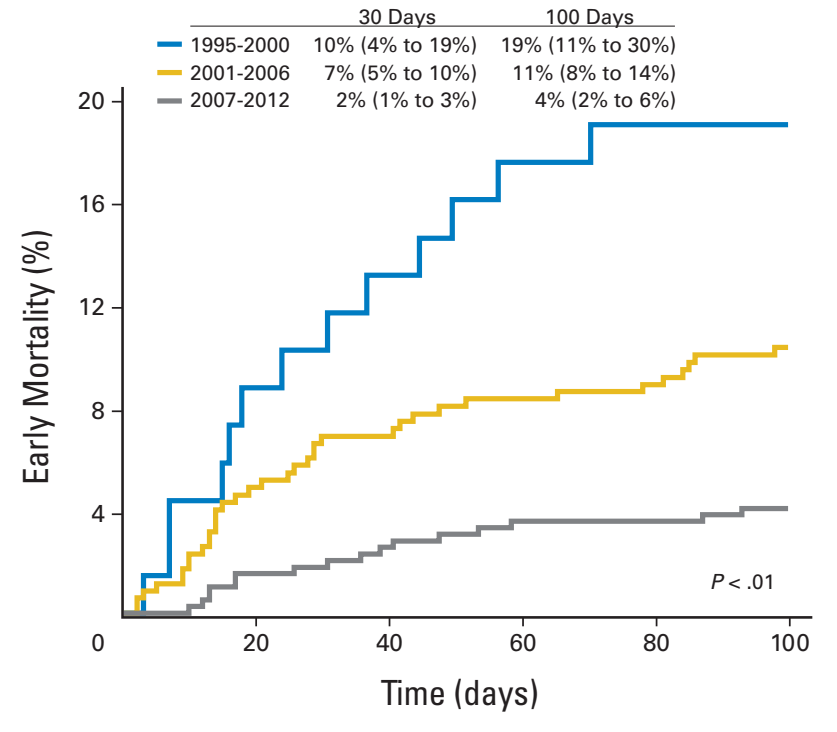

Fig 2. Trends in (A) overall survival (OS) for cardiac light-chain amyloidosis (AL); (B) OS for renal, noncardiac $A L$; $(C)$ early mortality (EM) based on center experience; and (D) time trends in improvement in EM among centers.

identified was 9 months after transplantation. During the initial period, no difference occurred between the 2001-to-2006 and 2007-to2012 cohorts for relapse and/or progression. After 9 months, the relapse and/or progression rate was lower in the 2007-to-2012 cohort. The presence of cardiac involvement was associated with increased EM (hazard ratio [HR], 3.5; 95\% CI, 2 to $6.4 ; P<.001$ ), worse PFS (HR, 2.0; 95\% CI, 1.7 to 3.0; $P<.001$ ), and OS (HR, 2.5; 95\% CI, 1.6 to $3.9 ; P<.001)$.

\section{DISCUSSION}

We describe outcomes after autotransplantation in the largest cohort of patients with AL reported to date. We make a number of clinically important observations. First, impressive reduction in EM has been achieved in recent years, and this is superior to the reported TRM of $24 \%$ from the only randomized clinical trial we know of in this setting. ${ }^{7}$ Second, cardiac amyloid involvement remains the critical vari- able associated with worse outcomes, with no improvement in outcomes of patients with cardiac amyloidosis over time. Third, center experience with AL transplantation is important in reducing EM. Centers that perform four or more AL transplantations per year report better results than those that do not. Fourth, 5-year survival in this real-world experience of autotransplantation has improved to $77 \%$ in the most recent cohort. This is comparable to data from specialized single centers and superior to the transplant arm of the randomized trial. ${ }^{7,14}$

Although established as an effective treatment modality in AL, autotransplantation has been controversial, owing to inferior outcomes reported in the prospective, randomized controlled, phase III trial described in 2007. ${ }^{7}$ Further, more recently, combination chemotherapy using novel agents such as bortezomib with alkylators has been shown to be associated with prompt and deep hematologic responses in the upfront setting. ${ }^{15-18}$ Therefore, the role of autotransplantation in AL has been further questioned. This is reflected in our 


\begin{tabular}{|c|c|c|c|c|c|}
\hline \multirow[b]{2}{*}{ Response } & \multicolumn{2}{|c|}{$\begin{array}{c}2001-2006 \\
(n=197)\end{array}$} & \multicolumn{2}{|c|}{$\begin{array}{c}2007-2012 \\
(n=157)\end{array}$} & \multirow[b]{2}{*}{$P$} \\
\hline & No. & $\%$ & No. & $\%$ & \\
\hline Best hematologic response & & & & & .005 \\
\hline Complete response & 60 & 30 & 58 & 37 & \\
\hline Partial response & 44 & 22 & 53 & 34 & \\
\hline Stable disease & 42 & 21 & 25 & 16 & \\
\hline Progression & 7 & 4 & 3 & 2 & \\
\hline Nonevaluable* & 22 & 11 & 14 & 9 & \\
\hline Missing & 22 & 11 & 4 & 3 & \\
\hline Best renal response & & & & & .30 \\
\hline Response & 61 & 31 & 51 & 32 & \\
\hline Stable disease & 48 & 24 & 47 & 30 & \\
\hline Progression & 7 & 4 & 10 & 6 & \\
\hline Nonevaluable* & 22 & 11 & 14 & 9 & \\
\hline Missing & 59 & 30 & 35 & 22 & \\
\hline
\end{tabular}

study; the number of transplantations performed in the US did not rise as rapidly after 2007 as they did in the preceding years.

Despite similar proportion of patients with cardiac involvement, transplantation after 2007 was associated with lower EM and superior OS than the earlier time periods. Therefore, EM improvements appeared to be multifactorial, representing improvements in supportive care and a higher level of experience overall in caring for these patients with complex conditions and not merely selection of fitter patients. Among patients with a renal, noncardiac pattern of AL involvement, outcomes improved significantly over time. However, for patients with cardiac AL, we were unable to demonstrate a statistically significant change in survival over time. A similar finding has been reported by others with novel chemotherapies ${ }^{17,19,20}$ and highlights the importance of earlier diagnosis of AL disease and an unmet need for safer and more effective therapies for patients with advanced cardiac amyloidosis, including antifibril therapies.

In our subset analyses of 354 patients, we were able to study response rates and prognostic factors. The response rate at day 100 was limited by missing data, particularly for the 2001-to-2006 group; therefore, it is not shown. However, when we assessed best hematologic response to transplantation, we noted more complete and partial hematologic responses in 2007 to 2012 than in 2001 to 2006. More patients received pretransplantation induction chemotherapy during 2007 to 2012, but melphalan conditioning doses were also lower in this period than in others. Of interest, renal response rates were equivalent in 2001 to 2006 and 2007 to 2012 at $31 \%$ and $32 \%$, respectively; this result is similar to renal response of 33\% reported after chemotherapy. ${ }^{21}$ However, fewer patients, less than $10 \%$, in both groups had progression of renal AL. This was much lower compared with renal progression after chemotherapy, even among the $50 \%$ of patients who have complete and partial hematologic responses. ${ }^{21}$

A number of pretransplantation factors have been shown to be prognostic after autotransplantation for AL. Cardiac biomarkers using serum cardiac troponin $\mathrm{T}$ and $\mathrm{N}$-terminal of brain natriuretic peptide provide a simple clinical prognostication system of staging $\mathrm{AL}$ amyloidosis, ${ }^{9}$ improving our ability to screen for patients with advanced cardiac amyloidosis who are at increased risk for post-

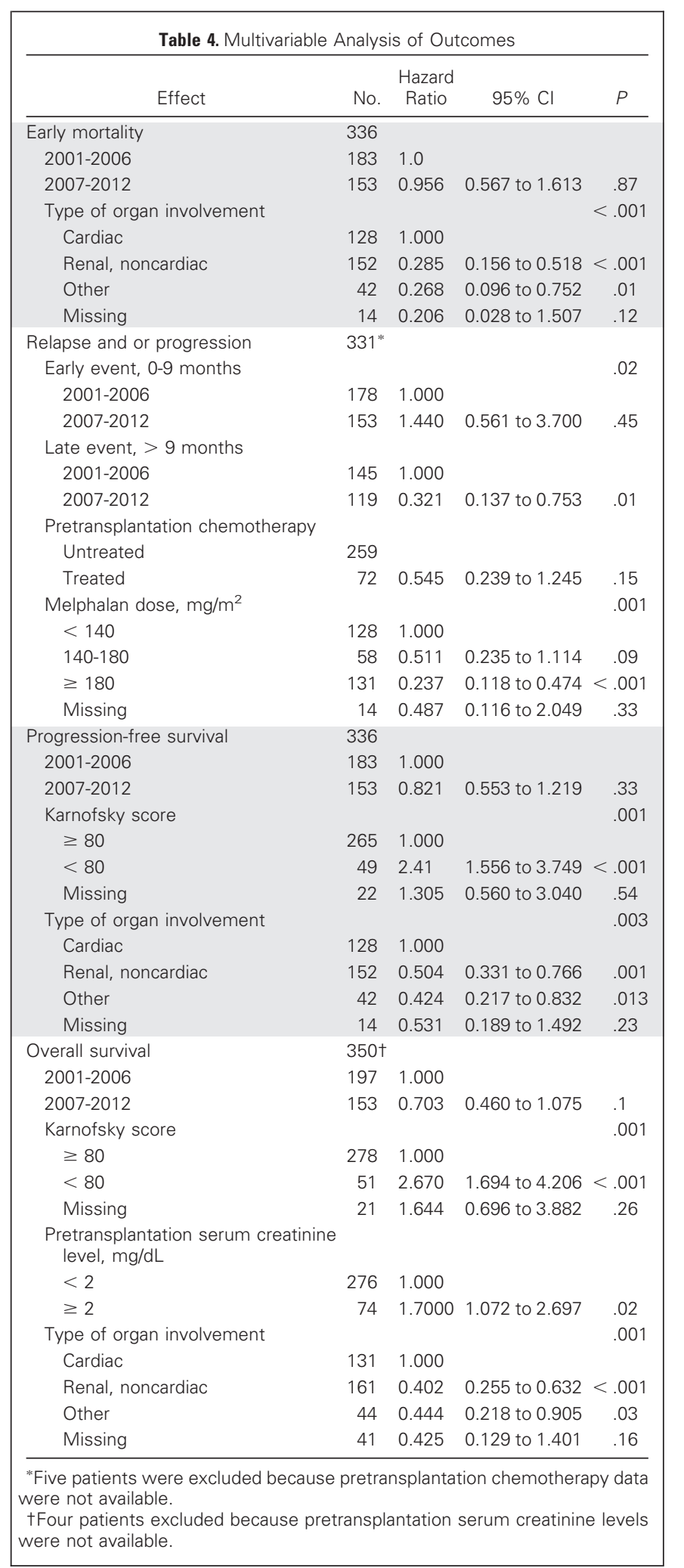

transplantation complications and EM. Other known risk factors associated with transplantation mortality include performance status and multiorgan involvement with amyloidosis. ${ }^{22,23}$ In addition, lowered melphalan conditioning intensity to adjust for risk has been 
associated with a reduced response rate. ${ }^{24}$ In this study, we confirm that cardiac involvement, poor renal function, poor KPS less than 80 , and use of melphalan conditioning less than $180 \mathrm{mg} / \mathrm{m}^{2}$ are associated with worse outcomes. The HCT-CI is a transplantationspecific prognostic variable that was described and validated in the mid-2000s. Although we were able to calculate it many patients using reported comorbidities, this variable was unavailable in the pre-2008 patient set. A bone marrow plasma cell clone greater than $10 \%$ has been found to be associated with worse outcomes, ${ }^{25,26}$ but this finding was not replicated in our study. Although pretransplantation chemotherapy was not associated with improved outcomes, most of the pretransplantation chemotherapy included nonbortezomib-based approaches.

In addition to the superior hematologic responses noted previously, patients in the 2007-to-2012 cohort also had a lower risk of relapse starting 9 months after transplantation. This was an interesting finding because lower doses of melphalan conditioning, which was more common in the 2007-to-2012 period that in others, was an independent predictor of relapse. Post-transplantation consolidation with bortezomib has been associated with deepening of response rates. $^{27}$ In our analysis, only 38 patients received any posttransplantation consolidation, and further analyses could not be performed because of low numbers.

The best results for autotransplantation in AL have been reported from specialized, high-volume centers in contrast to the multicenter, randomized trial experience. It has been proposed that center experience in treating patients with AL is important, with low-volume centers having higher EM rates. ${ }^{8}$ Indeed, in our cohort, transplantation centers performing fewer than four AL transplantations per year had higher EM rates than those of centers performing four or more transplantations. Lack of differences in the patient, organ, or chemotherapy-related factors between high- and low-volume centers led us to believe that high-volume centers do not necessarily select fitter patients for transplantation. Rather, they may be more experienced in supporting and treating these patients in the early posttransplantation period. However, owing to the small numbers in the subset analysis, our analysis was not powered to show significant differences in outcomes on the basis of center volume in the multivariable analysis after other factors were adjusted.

Our study had a number of limitations inherent to its retrospective nature and the long interval under study. Amyloidosis manage- ment is a dynamic field, and, during study inclusion, criteria for response and organ involvement changed twice. ${ }^{12,28}$ Variability in reporting by centers and the introduction of markers such as FLC, cardiac biomarkers, and HCT-CI at various points during this period were other issues inherent to the interval under study. Data regarding FLC and cardiac biomarkers were not collected in our database after 2008, but serial FLC or cardiac biomarkers were reported for too few patients to adequately define amyloid stage $\mathrm{e}^{29}$ or response by using the 2012 criteria described by Palladini et al. ${ }^{28}$ Another limitation of our study was our inability to stratify the severity of cardiac involvement because cardiac biomarker information was not uniformly reported. Therefore, although percentages of patients reported with cardiac involvement were similar between the 2001-to-2006 and 2007-to2012 periods, we do not know if cardiac stage was similar between these cohorts. Finally, our data were limited to individuals undergoing transplantation, who are inherently a selected group of better-risk patients. $^{30}$

Although our study does not replace a controlled, transplantationversus-nontransplantation trial, it is reassuring to see that excellent outcomes of autotransplantation previously reported from large single centers were replicated in practice capturing most AL transplantations in the US. With the availability of novel plasma cell-directed agents such as bortezomib, we propose that it is time to reconsider a multi-institutional study to compare transplantation, perhaps limited to high-volume centers, versus novel nontransplantation therapy.

\section{AUTHORS' DISCLOSURES OF POTENTIAL CONFLICTS} OF INTEREST

Disclosures provided by the authors are available with this article at www.jco.org.

\section{AUTHOR CONTRIBUTIONS}

Conception and design: Anita D’Souza, Angela Dispenzieri, Baldeep Wirk, Mei-Jie Zhang, Parameswaran Hari

Collection and assembly of data: Anita D'Souza, Angela Dispenzieri, Baldeep Wirk, Mei-Jie Zhang, Jiaxing Huang, Parameswaran Hari

Data analysis and interpretation: All authors

Manuscript writing: All authors

Final approval of manuscript: All authors

\section{REFERENCES}

1. Skinner $M$ : $A L$ amyloidosis: The last 30 years. Amyloid 7:13-14, 2000

2. Merlini G, Seldin DC, Gertz MA: Amyloidosis: Pathogenesis and new therapeutic options. J Clin Oncol 29:1924-1933, 2011

3. Comenzo RL, Reece D, Palladini G, et al: Consensus guidelines for the conduct and reporting of clinical trials in systemic light-chain amyloidosis. Leukemia 26:2317-2325, 2012

4. Dispenzieri A, Kyle RA, Lacy MQ, et al: Superior survival in primary systemic amyloidosis patients undergoing peripheral blood stem cell transplantation: A casecontrol study. Blood 103:3960-3963, 2004

5. Skinner M, Sanchorawala V, Seldin DC, et al: High-dose melphalan and autologous stem-cell transplantation in patients with $\mathrm{AL}$ amyloidosis: An 8-year study. Ann Intern Med 140:85-93, 2004
6. Mollee PN, Wechalekar AD, Pereira DL, et al: Autologous stem cell transplantation in primary systemic amyloidosis: The impact of selection criteria on outcome. Bone Marrow Transplant 33:271-277, 2004

7. Jaccard A, Moreau $P$, Leblond $V$, et al: High-dose melphalan versus melphalan plus dexamethasone for $\mathrm{AL}$ amyloidosis. N Engl J Med 357: 1083-1093, 2007

8. Mehta J: High-dose melphalan versus melphalan plus dexamethasone for $\mathrm{AL}$ amyloidosis. $\mathrm{N}$ Engl J Med 358:91, 2008

9. Dispenzieri A, Gertz MA, Kyle RA, et al: Serum cardiac troponins and $\mathrm{N}$-terminal pro-brain natriuretic peptide: A staging system for primary systemic amyloidosis. J Clin Oncol 22:3751-3757, 2004

10. Tsai SB, Seldin DC, Quillen K, et al: High-dose melphalan and stem cell transplantation for patients with $\mathrm{AL}$ amyloidosis: Trends in treatment-related mortality over the past 17 years at a single referral center. Blood 120:4445-4446, 2012

11. Gertz MA, Lacy MQ, Dispenzieri A, et al: Refinement in patient selection to reduce treatment-related mortality from autologous stem cell transplantation in amyloidosis. Bone Marrow Transplant 48:557-561, 2013

12. Gertz MA, Comenzo $R$, Falk $R H$, et al: Definition of organ involvement and treatment response in immunoglobulin light chain amyloidosis $(A L)$ : a consensus opinion from the 10th International Symposium on Amyloid and Amyloidosis, Tours, France, 18-22 April 2004. Am J Hematol 79:319-328, 2005

13. Horowitz MM, Przepiorka D, Champlin RE, et al: Should HLA-identical sibling bone marrow transplants for leukemia be restricted to large centers? Blood 79:2771-2774, 1992

14. Cibeira MT, Sanchorawala V, Seldin DC, et al: Outcome of $\mathrm{AL}$ amyloidosis after high-dose melphalan and autologous stem cell transplantation: 
Long-term results in a series of 421 patients. Blood 118:4346-4352, 2011

15. Mikhael JR, Schuster SR, Jimenez-Zepeda $\mathrm{VH}$, et al: Cyclophosphamide-bortezomibdexamethasone (CyBorD) produces rapid and complete hematologic response in patients with $\mathrm{AL}$ amyloidosis. Blood 119:4391-4394, 2012

16. Palladini G, Milani $P$, Foli $A$, et al: Melphalan and dexamethasone with or without bortezomib in newly diagnosed AL amyloidosis: A matched casecontrol study on 174 patients. Leukemia 28:23112316, 2014

17. Venner CP, Gillmore JD, Sachchithanantham $S$, et al: A matched comparison of cyclophosphamide, bortezomib and dexamethasone (CVD) versus risk-adapted cyclophosphamide, thalidomide and dexamethasone (CTD) in AL amyloidosis. Leukemia 28:2304-2310, 2014

18. Venner CP, Lane T, Foard D, et al: Cyclophosphamide, bortezomib, and dexamethasone therapy in $\mathrm{AL}$ amyloidosis is associated with high clonal response rates and prolonged progression-free survival. Blood 119:4387-4390, 2012

19. Merlini G, Palladini G: Treating advanced cardiac damage in light chain amyloidosis: Still an unmet need. Haematologica 99:1407-1409, 2014
20. Jaccard A, Comenzo RL, Hari $P$, et al: Efficacy of Bortezomib, Cyclophosphamide and Dexamethasone in treatment of naive patients with high risk cardiac AL amyloidosis (Mayo Clinic stage III). Haematologica, 99:1479-1485, 2014

21. Pinney $J H$, Lachmann $H J$, Bansi $L$, et al: Outcome in renal $\mathrm{Al}$ amyloidosis after chemotherapy. J Clin Oncol 29:674-681, 2011

22. Kumar SK, Gertz MA, Lacy $M Q$, et al: Recent improvements in survival in primary systemic amyloidosis and the importance of an early mortality risk score. Mayo Clin Proc 86:12-18, 2011

23. Dispenzieri A, Gertz MA, Buadi F: What do I need to know about immunoglobulin light chain (AL) amyloidosis? Blood Rev 26:137-154, 2012

24. Gertz MA, Lacy MQ, Dispenzieri $A$, et al: Risk-adjusted manipulation of melphalan dose before stem cell transplantation in patients with amyloidosis is associated with a lower response rate. Bone Marrow Transplant 34:1025-1031, 2004

25. Dinner S, Witteles W, Witteles $R$, et al: The prognostic value of diagnosing concurrent multiple myeloma in immunoglobulin light chain amyloidosis. Br J Haematol 161:367-372, 2013

26. Kourelis TV, Kumar SK, Gertz MA, et al: Coexistent multiple myeloma or increased bone mar- row plasma cells define equally high-risk populations in patients with immunoglobulin light chain amyloidosis. J Clin Oncol 31:4319-4324, 2013

27. Landau $H$, Hassoun $H$, Rosenzweig MA, et al: Bortezomib and dexamethasone consolidation following risk-adapted melphalan and stem cell transplantation for patients with newly diagnosed light-chain amyloidosis. Leukemia 27:823-828, 2013

28. Palladini G, Dispenzieri A, Gertz $M A$, et al: New criteria for response to treatment in immunoglobulin light chain amyloidosis based on free light chain measurement and cardiac biomarkers: Impact on survival outcomes. J Clin Oncol 30:4541-4549, 2012

29. Kumar S, Dispenzieri A, Lacy MQ, et al: Revised prognostic staging system for light chain amyloidosis incorporating cardiac biomarkers and serum free light chain measurements. J Clin Oncol 30:989-995, 2012

30. Dispenzieri A, Lacy $M Q$, Kyle RA, et al: Eligibility for hematopoietic stem-cell transplantation for primary systemic amyloidosis is a favorable prognostic factor for survival. J Clin Oncol 19:3350-3356, 2001

\section{Affiliations}

Anita D'Souza, Mei-Jie Zhang, Jiaxing Huang, and Parameswaran Hari, Center for International Blood and Marrow Transplant Research, Medical College of Wisconsin; Mei-Jie Zhang, Institute for Health and Society, Medical College of Wisconsin, Milwaukee; Natalie S. Callander, University of Wisconsin Carbone Cancer Center, Madison, WI; Angela Dispenzieri, Morie A. Gertz, Robert A. Kyle, and Shaji Kumar, Mayo Clinic, Rochester, MN; Baldeep Wirk, Seattle Cancer Care Alliance, Seattle, WA; Raymond L. Comenzo, Tufts Medical Center, Boston; Muthalagu Ramanathan, University of Massachusetts Memorial Medical Center, Worcester, MA; Robert Peter Gale, Hematology Research Centre, Imperial College London, London, United Kingdom; Hillard M. Lazarus, Seidman Cancer Center, University Hospitals Case Medical Center, Cleveland, OH; Bipin N. Savani and Robert F. Cornell, Vanderbilt University Medical Center, Nashville, TN; Brendan M. Weiss and Dan T. Vogl, Abramson Cancer Center, University of Pennsylvania Medical Center, Philadelphia, PA; César O. Freytes, South Texas Veterans Health Care System and University of Texas Health Science Center San Antonio, San Antonio; Rammurti T. Kamble, Center for Cell and Gene Therapy, Baylor College of Medicine, Houston, TX; Emma C. Scott, Center for Hematologic Malignancies, The Knight Cancer Institute, Oregon Health and Science University, Portland, OR; Heather J. Landau, Memorial Sloan Kettering Cancer Center; Tomer M. Mark, Weill Cornell Medical College, New York, NY; Jan S. Moreb, Shands HealthCare and University of Florida, Gainesville; Taiga Nishihori, H. Lee Moffitt Cancer Center and Research Institute, Tampa, FL; Luciano J. Costa, University of Alabama at Birmingham, Birmingham, AL; Richard F. Olsson, Karolinska Institutet, Stockholm; Centre for Clinical Research Sörmland, Uppsala University, Uppsala, Sweden; Siddhartha Ganguly, University of Kansas Medical Center, Kansas City, KS; Tamila L. KindwallKeller, University of Virginia Health System, Charlottesville, VA; and William A. Wood, University of North Carolina, Chapel Hill, NC.

\section{Support}

Supported by Public Health Service Grant/Cooperative Agreement No. U24-CA076518 from the National Cancer Institute (NCI), the National Heart, Lung and Blood Institute (NHLBI), and the National Institute of Allergy and Infectious Diseases (NIAID); Grant/Cooperative Agreement No. 5U10HL069294 from the NHLBI and the NCI; contract No. HHSH250201200016C with Health Resources and Services Administration (HRSA/ DHHS); Grants No. N00014-13-1-0039 and No. N00014-14-1-0028 from the Office of Naval Research; and grants from Actinium Pharmaceuticals; Allos Therapeutics; Amgen; an anonymous donation to the Medical College of Wisconsin; Ariad; Be the Match Foundation; Blue Cross and Blue

Shield Association; Celgene; Chimerix; Fred Hutchinson Cancer Research Center; Fresenius-Biotech North America; Gamida Cell Teva Joint Venture; Genentech; Gentium SpA; Genzyme Corporation; GlaxoSmithKline; Health Research Roswell Park Cancer Institute; HistoGenetics; Incyte Corporation; Jeff Gordon Children's Foundation; Kiadis Pharma; The Leukemia \& Lymphoma Society; Medac; The Medical College of Wisconsin; Merck \& Co; Millennium: The Takeda Oncology Co.; Milliman USA; Miltenyi Biotec; the National Marrow Donor Program; Onyx Pharmaceuticals; Optum Healthcare Solutions; Osiris Therapeutics; Otsuka America Pharmaceutical; Perkin Elmer; Remedy Informatics; Sanofi US; Seattle Genetics;

Sigma-Tau Pharmaceuticals; Soligenix; St. Baldrick's Foundation; StemCyte, A Global Cord Blood Therapeutics Co.; Stemsoft Software; Swedish Orphan Biovitrum; Tarix Pharmaceuticals; TerumoBCT; Teva Neuroscience; THERAKOS; the University of Minnesota; the University of Utah; and Wellpoint (CIBMTR). Supported by the National Center for Research Resources and the National Center for Advancing Translational Sciences, National Institutes of Health, Grant No. 8UL1TR000055 (D'Souza). 


\section{AUTHORS' DISCLOSURES OF POTENTIAL CONFLICTS OF INTEREST}

Improved Outcomes After Autologous Hematopoietic Cell Transplantation for Light Chain Amyloidosis: A Center for International Blood and Marrow Transplant Research Study

The following represents disclosure information provided by authors of this manuscript. All relationships are considered compensated. Relationships are self-held unless noted. I = Immediate Family Member, Inst = My Institution. Relationships may not relate to the subject matter of this manuscript. For more information about ASCO's conflict of interest policy, please refer to www.asco.org/rwc or jco.ascopubs.org/site/ifc.

\section{Anita D'Souza}

No relationship to disclose

\section{Angela Dispenzieri}

Consulting or Advisory Role: Prothena (Inst), Pfizer (Inst)

Research Funding: Celgene (Inst), Millennium Pharmaceuticals (Inst), Janssen Oncology (Inst), Pfizer (Inst)

Travel, Accommodations, Expenses: Pfizer

Baldeep Wirk

No relationship to disclose

Mei-Jie Zhang

No relationship to disclose

\section{Jiaxing Huang}

No relationship to disclose

\section{Morie A. Gertz}

Honoraria: Celgene, Novartis, Millenium Pharmaceuticals, Med Learning Group, Research to Practice, Onyx Pharmaceuticals, ISIS, Sanofi, Prothena

\section{Robert A. Kyle}

No relationship to disclose

\section{Shaji Kumar}

Consulting or Advisory Role: Skyline Diagnostics

Research Funding: Celgene, Novartis, Onyx, Millennium, Sanofi

Pasteur, Janssen Pharmaceuticals

\section{Raymond L. Comenzo}

Consulting or Advisory Role: Prothena, Takeda, Jannsen

Research Funding: Prothena, Takeda Pharmaceuticals, Jannsen

Pharmaceuticals, Array BioPharma, Karyopharm

\section{Robert Peter Gale}

Employment: Celgene

Stock or Other Ownership: Celgene

Travel, Accommodations, Expenses: Celgene

Hillard M. Lazarus

Honoraria: Celgene

Speakers' Bureau: Celgene

Travel, Accommodations, Expenses: Celgene

Bipin N. Savani

No relationship to disclose

Robert F. Cornell

No relationship to disclose

\section{Brendan M. Weiss}

Research Funding: Prothena, Janssen Research \& Development

Dan T. Vogl

Consulting or Advisory Role: Celgene, Amgen, Millennium Pharmaceuticals

Research Funding: Millennium Pharmaceuticals, Acetylon

Pharmaceuticals, GlaxoSmithKline, Calithera Biosciences, Constellation Pharmaceuticals

César O. Freytes

Consulting or Advisory Role: Spectrum Pharmaceuticals, Clinipace

Speakers' Bureau: Sanofi

Research Funding: Merck
Emma C. Scott

Honoraria: Takeda, Onyx, Takeda (I), Onyx (I)

Consulting or Advisory Role: Millennium Pharmaceuticals, Onyx Research Funding: Millennium Pharmaceuticals, Takeda

Heather J. Landau

Honoraria: Takeda, Prothena, Onyx, Spectrum Pharmaceuticals

Consulting or Advisory Role: Prothena

Research Funding: Onyx, Takeda

Jan S. Moreb

No relationship to disclose

Luciano J. Costa

No relationship to disclose

Muthalagu Ramanathan

Employment: Zoll (I)

Stock or Other Ownership: Smith \& Nephew (I)

Consulting or Advisory Role: Med Learning Group

Research Funding: Celgene, Gilead Sciences, Millennium

Pharmaceuticals, Pharmacyclics

Travel, Accommodations, Expenses: Gilead Sciences

Natalie S. Callander

No relationship to disclose

Rammurti T. Kamble

No relationship to disclose

Richard F. Olsson

No relationship to disclose

Siddhartha Ganguly

Honoraria: Seattle Genetics, Onyx

Consulting or Advisory Role: Takeda, Millennium, ARIAD

Speakers' Bureau: Onyx, Seattle Genetics

Research Funding: Janssen Pharmaceuticals, Sanofi, Celgene

Travel, Accommodations, Expenses: Onyx, Seattle Genetics

Taiga Nishihori

No relationship to disclose

Tamila L. Kindwall-Keller

Research Funding: Celldex, US Biotest

William A. Wood

No relationship to disclose

Tomer M. Mark

Stock or Other Ownership: Abbvie, Karyopharm Therapeutics, Portola Pharmaceuticals

Consulting or Advisory Role: Celgene, Janssen Pharmaceuticals, Takeda Speakers' Bureau: Celgene, Takeda, Onyx

Research Funding: Celgene (Inst), Onyx (Inst)

Parameswaran Hari

Stock or Other Ownership: Pharmacyclics

Honoraria: Celgene, Millennium, Sanofi, Onyx

Consulting or Advisory Role: Celgene, Millennium Pharmaceuticals, Onyx, Sanofi

Research Funding: Millennium Pharmaceuticals (Inst), Celgene (Inst) 


\section{Appendix}

\begin{tabular}{|c|c|c|c|}
\hline \multicolumn{4}{|l|}{ Age at transplantation, years } \\
\hline $18-39$ & $11(3)$ & $44(3)$ & \\
\hline $40-49$ & $62(18)$ & $242(17)$ & \\
\hline $50-59$ & $146(41)$ & $539(39)$ & \\
\hline Sex & & & .73 \\
\hline Male & $216(61)$ & $838(60)$ & \\
\hline Female & $138(39)$ & $558(40)$ & \\
\hline Country & & & .08 \\
\hline United States & $329(93)$ & $1,330(95)$ & \\
\hline Canada & $25(7)$ & $66(5)$ & \\
\hline Missing & $9(3)$ & $47(3)$ & \\
\hline Karnofsky score* & & & .02 \\
\hline $80-100$ & $278(79)$ & $1,031(74)$ & \\
\hline$<80$ & $51(14)$ & $192(14)$ & \\
\hline Missing & $25(7)$ & $173(12)$ & \\
\hline Time from diagnosis to transplantation, months & & & .56 \\
\hline$<6$ & $234(66)$ & $882(63)$ & \\
\hline $6-12$ & $82(23)$ & $359(26)$ & \\
\hline $12-24$ & $38(11)$ & $155(11)$ & \\
\hline Median follow-up of survivors (range), months & $61(3-145)$ & $47(3-145)$ & \\
\hline
\end{tabular}

\begin{tabular}{|c|c|c|c|c|}
\hline \multirow[b]{2}{*}{ Outcome } & \multicolumn{2}{|c|}{ Research Data Set $(n=354)$} & \multicolumn{2}{|c|}{ Entire Cohort $(N=1,396)$} \\
\hline & Probability (\%) & $95 \% \mathrm{Cl}$ & Probability (\%) & $95 \% \mathrm{Cl}$ \\
\hline \multicolumn{5}{|l|}{ Early mortality } \\
\hline 1 month & 94 & 91 to 96 & 96 & 95 to 97 \\
\hline 100 day & 90 & 86 to 93 & 93 & 91 to 94 \\
\hline 3 & 76 & 71 to 80 & 77 & 75 to 80 \\
\hline 5 & 70 & 64 to 75 & 68 & 65 to 71 \\
\hline
\end{tabular}


D'Souza et al

\begin{tabular}{|c|c|c|c|c|c|}
\hline \multirow[b]{3}{*}{ Variable } & \multicolumn{4}{|c|}{ Center Experience, No. of Transplantations } & \multirow[b]{3}{*}{$P$} \\
\hline & \multicolumn{2}{|c|}{$<4(\mathrm{n}=91)$} & \multicolumn{2}{|c|}{$\geq 4(n=66)$} & \\
\hline & No. & $\%$ & No. & $\%$ & \\
\hline Age at transplantation, years & & & & & .60 \\
\hline $18-39$ & 3 & 3 & 5 & 8 & \\
\hline $40-49$ & 13 & 14 & 6 & 9 & \\
\hline $50-59$ & 40 & 44 & 27 & 41 & \\
\hline $60-65$ & 19 & 21 & 17 & 26 & \\
\hline$\geq 66$ & 16 & 18 & 11 & 17 & \\
\hline Karnofsky score & & & & & .68 \\
\hline $80-100$ & 71 & 78 & 52 & 79 & \\
\hline$<80$ & 13 & 14 & 7 & 11 & \\
\hline Missing & 7 & 8 & 7 & 11 & \\
\hline Time from diagnosis to transplantation, months & & & & & .49 \\
\hline$<6$ & 63 & 69 & 40 & 61 & \\
\hline $6-12$ & 18 & 20 & 18 & 27 & \\
\hline $12-24$ & 10 & 11 & 8 & 12 & \\
\hline Cardiac involvement & & & & & .24 \\
\hline Yes & 37 & 41 & 22 & 33 & \\
\hline No & 32 & 35 & 32 & 48 & \\
\hline Missing & 22 & 24 & 12 & 18 & \\
\hline Renal involvement & & & & & .65 \\
\hline Yes & 68 & 75 & 52 & 79 & \\
\hline No & 11 & 12 & 5 & 8 & \\
\hline Missing & 12 & 13 & 9 & 14 & \\
\hline Organ involvement & & & & & .13 \\
\hline 0 & 1 & 1 & 2 & 3 & \\
\hline 1 & 29 & 32 & 23 & 35 & \\
\hline 2 & 36 & 40 & 15 & 23 & \\
\hline 3 & 18 & 20 & 15 & 23 & \\
\hline 4 & 7 & 8 & 11 & 17 & \\
\hline Hematopoietic cell transplantation-comorbidity index & & & & & .15 \\
\hline 0 & 25 & 27 & 8 & 12 & \\
\hline 1 & 10 & 11 & 9 & 14 & \\
\hline 2 & 13 & 14 & 14 & 21 & \\
\hline 3 & 13 & 14 & 7 & 11 & \\
\hline 4 & 24 & 26 & 19 & 29 & \\
\hline Missing & 6 & 7 & 9 & 14 & \\
\hline Melphalan dose, $\mathrm{mg} / \mathrm{m}^{2}$ & & & & & .20 \\
\hline$<140$ & 35 & 38 & 34 & 52 & \\
\hline $140-180$ & 18 & 20 & 13 & 20 & \\
\hline$>180$ & 37 & 41 & 17 & 26 & \\
\hline Missing & 1 & 1 & 2 & 3 & \\
\hline Chemotherapy & & & & & .09 \\
\hline No chemotherapy & 65 & 71 & 40 & 61 & \\
\hline Melphalan based & 6 & 7 & 9 & 14 & \\
\hline Thalidomide based & 2 & 2 & 7 & 11 & \\
\hline Lenalidomide based & 9 & 10 & 4 & 6 & \\
\hline Bortezomib based & 9 & 10 & 6 & 9 & \\
\hline
\end{tabular}

